\title{
Artificial Molecular Machines Powered by Light
}

\author{
Matteo Amelia, Monica Semeraro, Serena Silvi, and Alberto Credi§* \\ §Grammaticakis-Neumann Prize Winner 2007
}

\begin{abstract}
The bottom-up construction and operation of mechanical machines of molecular size is a topic of great interest for nanoscience, and a fascinating challenge of nanotechnology. Like their macroscopic counterparts, nanoscale machines need energy to operate. Although most molecular motors of the biological world are fueled by chemical reactions, light is a very good choice to power artificial molecular machines because it can also be used to monitor the state of the machine, and allows systems to be obtained that show autonomous operation and do not generate waste products. By adopting an incrementally staged design strategy, photoinduced processes can be engineered within rotaxane-type structures with the purpose of obtaining light-powered molecular machines. In this short review we will illustrate such an approach by describing some systems investigated in our laboratories.
\end{abstract}

Keywords: Molecular device $\cdot$ Nanoscience $\cdot$ Photochemistry $\cdot$ Rotaxane $\cdot$ Supramolecular chemistry

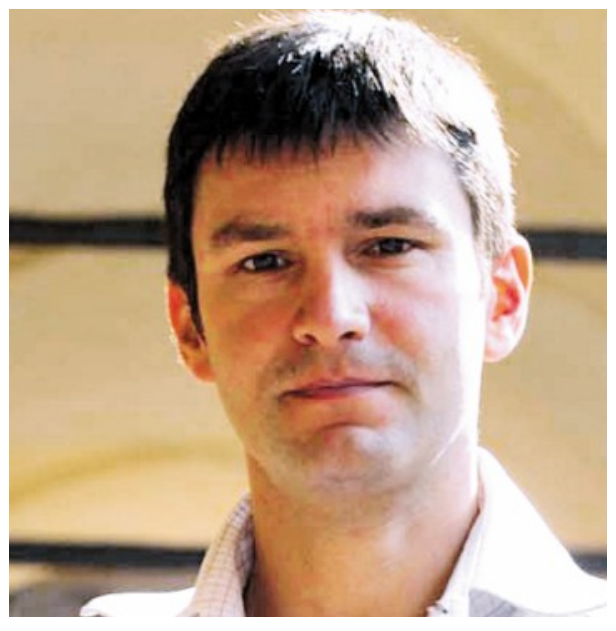

Alberto Credi is Professor of General and Inorganic Chemistry at the University of Bologna. Born in 1970, he gained his chemistry degree from the University of Bologna in 1994, where, after a research period at the University of Virginia, USA,

${ }^{*}$ Correspondence: Prof. Dr. A. Credi

Dipartimento di Chimica "G. Ciamician"

Università di Bologna

Via Selmi 2

40126 Bologna, Italy

Tel.: +390512099540

Fax: +390512099456

E-mail: alberto.credi@unibo.it he also earned his $\mathrm{PhD}$ in chemical sciences in 1999, under the supervision of Professor Vincenzo Balzani. He was then appointed researcher at the Faculty of Sciences of his Alma Mater, becoming Associate Professor in 2005. Prof. Credi has won many prizes, including the IUPAC Prize for Young Chemists (2000), and the Vincenzo Caglioti award of the Accademia Nazionale dei Lincei (2002). He is the co-author of more than 140 scientific papers in the fields of molecular and supramolecular photochemistry and electrochemistry, of a monograph entitled 'Molecular Devices and Machines' (together with V. Balzani and M. Venturi), and of a handbook of photochemistry.

\section{Introduction}

\subsection{Natural and Artificial \\ Nanomachines}

Nowadays the miniaturization race leads scientists to investigate the possibility of designing and constructing machines and motors at the nanometer scale, that is, at the molecular level. Many fields of technology, in particular information processing, have benefited from progressive miniaturization of the components of devices in the last fifty years. A common prediction is that further progress in miniaturization will not only decrease the size and increase the power of computers, ${ }^{[1]}$ but could also open the way to new technologies in the fields of medicine, environment, energy and materials. ${ }^{[2]}$

A molecular machine can be defined ${ }^{[3]}$ as an assembly of a discrete number of molecular components (that is, a supramolecular structure) designed to perform specific mechanical movements as a consequence of appropriate external stimuli. The extension of the concept of machine to the molecular level is important not only for the sake of basic research, but also for the growth of nanoscience and the development of a bottom-up approach to nanotechnology. Chemists, by the nature of their discipline, are already at the bottom, since they are able to manipulate molecules (i.e. the smallest entities with distinct shapes and properties) and are therefore in the ideal position to develop bottom-up strategies for the construction of nanoscale machines.

Much of the inspiration to construct molecular devices and machines comes from the outstanding progress of molecular biology that has begun to reveal the secrets of the natural nanomachines which constitute the material basis of life. ${ }^{[4]}$ Surely, the supramolecular architectures of the biological world are themselves the premier, proven examples of the feasibility and utility of nanotechnology, and constitute a sound rationale for attempting the realization of artificial molecular devices. ${ }^{[5,6]}$ The bottom-up construction of machines as complex as those present in Nature is a prohibitive task.

Therefore chemists have tried i) to construct much simpler systems, without 
mimicking the complexity of the biological structures, ii) to understand the principles and processes at the basis of their operation, and iii) to investigate the challenging problems posed by interfacing artificial molecular machines with the macroscopic world, particularly as far as energy supply and information exchange are concerned.

In the last few years the development of powerful synthetic methodologies, combined with a device-driven ingenuity evolved from the attention to functions and reactivity, have led to remarkable achievements in this field. Several excellent reviews $^{[7-19]}$ and a monograph ${ }^{[3]}$ dealing with artificial molecular machines and motors are available.

\subsection{Basic Features of Molecular Machines}

Molecular machines operate through some kind of chemical reaction. Like macroscopic machines, they are characterized by i) the kind of energy input supplied to make them work, ii) the type of movement performed by their components, iii) the way in which their operation can be monitored, iv) the possibility to repeat the operation at will, thus establishing a cyclic process, v) the time scale needed to complete a cycle of operation, and vi) the function that can be ultimately carried out.

It should be recalled, however, that nanoscale machines cannot be simply considered as 'shrunken' versions of macroscopic counterparts because several intrinsic properties of molecular-level entities are quite different from those of macroscopic objects. ${ }^{[6]}$

Of the above list, point $\mathrm{i}$ - namely, the energy supply of the molecular machine is certainly the most significant. The obvious way to provide a chemical system with energy is through an exoergonic chemical reaction. This is what happens in our body, where the chemical energy supplied by food is stored in ATP molecules and used to power the biological machines that sustain life. ${ }^{[4-6]}$ If a molecular machine has to work by inputs of chemical energy, it will need addition of fresh reactants ('fuel') at any step of its working cycle, with the concomitant formation of waste products. Accumulation of such waste products will compromise the cyclic operation of the device unless they are removed from the system, as it happens in our body as well as in macroscopic internal combustion engines. In any case, since a molecular machine has to work by repeating cycles (point iv), a fundamental requirement is that any chemical process taking place in the system has to be reversible.

Nature, however, shows that in green plants the energy needed to sustain the machinery of life is supplied by sunlight. Photochemical energy inputs can indeed cause the occurrence of endoergonic chemical reactions, which can make a machine work without formation of waste products. Furthermore, light stimulation can be switched on and off easily and rapidly, and lasers provide the opportunity of working in small space and very short time domains. It should also be noted that light energy can be supplied to a molecule without physically 'touching' it, whereas in order to make use of electrical energy a chemical system needs to be connected to a circuit (e.g. an electrochemical cell).

In order to control and monitor the machine operation (point iii), the motion of the component parts should cause readable changes in some chemical or physical property of the system. Photochemical methods are very useful in this regard because photons are at the same time quanta of energy and bits of information and can play the dual role of 'writing' (i.e. causing a change in the system) and 'reading' (i.e. reporting the state of the system). ${ }^{[20]}$ Luminescence spectroscopy, in particular, is a valuable technique since it is easily accessible and offers good sensitivity and selectivity, along with the possibility of time-resolved studies.

An important property of molecular machines, related to energy supply and cyclic operation, is their capability to exhibit an autonomous behavior; that is, to keep operating, in a constant environment and without the intervention of an external operator, as long as the energy source is available. ${ }^{[3]}$ Natural motors are autonomous, ${ }^{[4]}$ but most of the artificial systems reported so far are not autonomous because, after the mechanical movement induced by a given input, they need another, opposite input to reset. Obviously, the operation of a molecular machine is accompanied by partial degradation of free energy into heat, regardless of the nature of the energy input.

The use of light to power nanoscale devices is relevant for another important reason. If and when a nanotechnology-based industry will be developed, its products will have to be powered by renewable energy sources, because it has become clear that the problem of energy supply is a crucial one for human civilization for the years ahead.[21] In this frame, the construction of nanodevices, including natural-artificial hybrids, ${ }^{[22]}$ that harness solar energy in the form of visible or near-UV light is an important possibility.

\subsection{Rotaxane-type Structures as Nanoscale Machines}

In principle, artificial molecular machines can be designed starting from several kinds of molecular and supramolecular systems, ${ }^{[3,7-11,14-19]}$ including DNA. ${ }^{[12,13]}$ However, for the reasons mentioned below, most of the systems constructed so far are based on interlocked molecular species such as rotaxanes and related species. The name of these compounds derives from the
Latin words rota and axis for wheel and axle. Rotaxanes ${ }^{[23]}$ are minimally composed (Fig. 1) of a dumbbell-shaped molecule surrounded by a macrocyclic compound (the 'ring') and terminated by bulky groups ('stoppers') that prevent disassembly. Important features of these systems derive from noncovalent interactions between components that contain complementary recognition sites. Such interactions, that are also responsible for the efficient templatedirected syntheses of rotaxanes, include electron donor-acceptor ability, hydrogen bonding, hydrophobic-hydrophylic character, $\pi-\pi$ stacking, electrostatic forces and, on the side of the strong interaction limit, metal-ligand bonding.

Rotaxanes are appealing systems for the construction of molecular machines because i) the mechanical bond allows a large variety of mutual arrangements of the molecular components, while conferring stability to the system, ii) the interlocked architecture limits the amplitude of the intercomponent motion in the three directions, iii) the stability of a specific arrangement (co-conformation) is determined by the strength of the intercomponent interactions, and iv) such interactions can be modulated by external stimulation.

Two interesting molecular motions can be envisaged in rotaxanes, namely i) translation, i.e. shuttling, of the ring along the axle (Fig. 1a), and ii) rotation of the ring around the axle (Fig. 1b).

Hence, rotaxanes are good prototypes for the construction of both linear and rotary molecular machines. Systems of type i, termed molecular shuttles, constitute indeed the most common implementation of the molecular machine concept with rotaxanes.

In the next section we will make use of a few examples taken from our own research to discuss how photoinduced processes can be engineered within rotaxane-type multi-

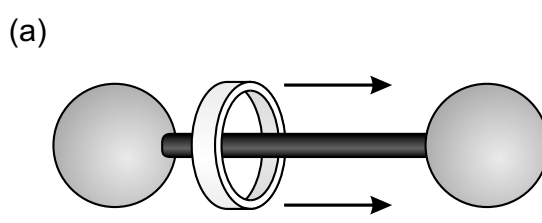

(b)

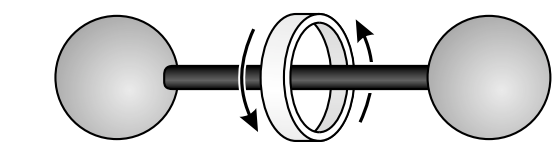

Fig. 1. Schematic representation of the principal intercomponent motions that can be obtained with rotaxanes: (a) shuttling and (b) ring rotation 
component molecular structures with the purpose of obtaining light-driven molecular machines.

\section{Results and Discussion}

\subsection{Molecular Shuttles Based on Photoinduced Electron Transfer}

Photoinduced electron-transfer reactions are of primary importance both in natural photosynthetic devices and in artificial systems. ${ }^{[24]}$ The first attempts ${ }^{[25,26]}$ aimed at exploiting photoinduced electron transfer to bring about large-amplitude controllable molecular motions involved the light-driven threading-dethreading of pseudorotaxanetype complexes. ${ }^{[27-30]}$

On the basis of the experience gained with some of these model systems, ${ }^{[28]}$ the bistable rotaxane $\mathbf{1}^{6+}$ (Fig. 2) was specifically designed ${ }^{[31]}$ to achieve photoinduced ring shuttling in solution. This compound has a modular structure; its ring component $\mathrm{R}$ is a $\pi$-electron-donating bis- $p$-phenylene-34-crown-10, whereas its dumbbell component is made of several covalently linked units. They are a $\mathrm{Ru}(\mathrm{II})$ polypyridine complex $\left(\mathrm{P}^{2+}\right)$, a $p$-terphenyl-type rigid spacer (S), a 4,4'-bipyridinium $\left(\mathrm{A}_{1}{ }^{2+}\right)$ and a 3,3'-dimethyl-4,4'-bipyridinium $\left(\mathrm{A}_{2}{ }^{2+}\right)$ $\pi$-electron-accepting stations, and a tetraarylmethane group as the terminal stopper (T). The Ru-based unit plays the dual role of a light-fueled power station and a stopper, whereas the mechanical switch consists of the two electron-accepting stations and the electron-donating macrocycle. Six $\mathrm{PF}_{6}{ }^{-}$ ions are present as the counteranions of the positively charged rotaxane. The stable translational isomer of rotaxane $\mathbf{1}^{6+}$ is the one in which the $\mathrm{R}$ component encircles the $\mathrm{A}_{1}{ }^{2+}$ unit, in keeping with the fact that this station is a better electron acceptor than the other one.

The strategy devised in order to obtain the photoinduced shuttling movement of $\mathrm{R}$ between the two stations $\mathrm{A}_{1}{ }^{2+}$ and $\mathrm{A}_{2}{ }^{2+}$ is based on a 'four stroke' synchronized sequence of electron-transfer and molecular rearrangement processes, as illustrated in Fig. 2. ${ }^{[31,32]}$ Light excitation of the photoactive unit $\mathrm{P}^{2+}$ (process 1 ) is followed by the transfer of an electron from this unit to $\mathrm{A}_{1}{ }^{2+}$ (process 2 ) which competes with the intrinsic decay of the $\mathrm{P}^{2+}$ excited state (process 3 ). After the reduction of $\mathrm{A}_{1}^{2+}$, with the consequent 'deactivation' of this station, the ring moves (process 4 ) by $1.3 \mathrm{~nm}$ towards $\mathrm{A}_{2}{ }^{2+}$, a step that is in competition with the back electron-transfer from $\mathrm{A}_{1}{ }^{+}$ (still encircled by $\mathrm{R}$ ) to the oxidized unit $\mathrm{P}^{3+}$ (process 5). Eventually, a back electrontransfer from the 'free' reduced station $\mathrm{A}_{1}{ }^{+}$ to the oxidized unit $\mathrm{P}^{3+}$ (process 6 ) restores the electron acceptor power to this radical cationic station. As a consequence of the

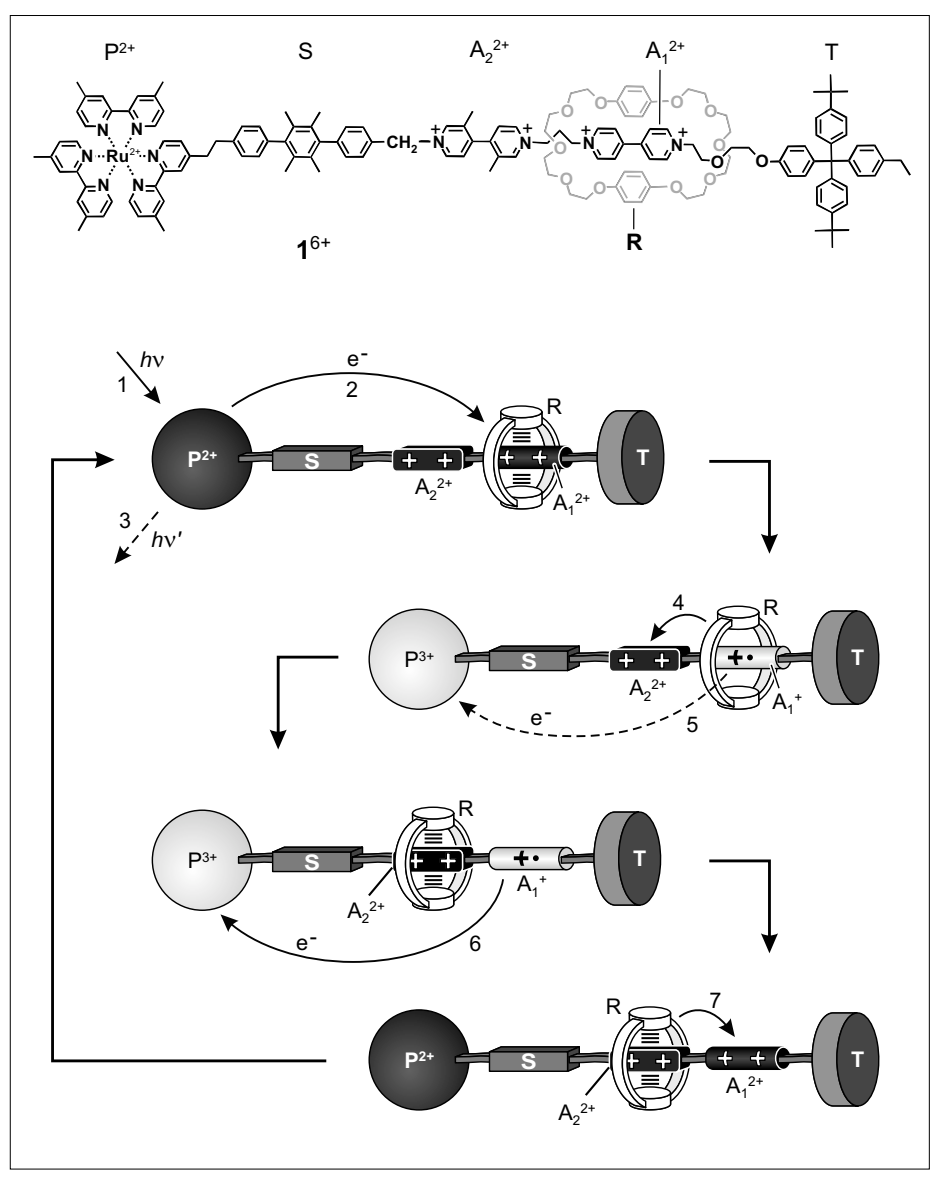

Fig. 2. Structure formula of rotaxane $1^{6+}$ and schematic representation of its operation as an autonomous molecular shuttle powered by visible light

electronic reset, thermally activated back movement of the ring from $\mathrm{A}_{2}{ }^{2+}$ to $\mathrm{A}_{1}{ }^{2+}$ takes place (process 7)

By means of steady-state and time-resolved spectroscopic experiments complemented by electrochemical measurements in acetonitrile solution, we showed[32] that the absorption of a visible photon by $\mathbf{1}^{6+}$ can cause the occurrence of a forward and back ring movement, that is, a full mechanical cycle according to the mechanism illustrated in Fig. 2. [33] The key issues of this mechanism are the competition between processes 2 (photoinduced electron transfer) and 3 (intrinsic excited-state deactivation), and between processes 4 (ring displacement from the reduced $\mathrm{A}_{1}$ station) and 5 (back electron transfer). From luminescence measurements we found ${ }^{[31]}$ that the time constants of processes 2 and 3 at $303 \mathrm{~K}$ are 4.0 and 0.9 $\mu \mathrm{s}$, respectively, corresponding to a quantum yield of 0.16 for the electron-transfer process. Transient absorption experiments allowed us to determine ${ }^{[31,32]}$ the time constants of processes 4 and 5, which are 47 and $6.7 \mu \mathrm{s}$, respectively, at $303 \mathrm{~K}$. Hence, the efficiency of ring displacement from the photo-reduced $\mathrm{A}_{1}$ station amounts to 0.12 ; because all the successive processes have no competitors, the overall shuttling quantum yield is simply $0.16 \times 0.12=0.02$. This somewhat disappointing figure is compen- sated by the fact that the investigated system gathers together the following features: i) it is powered by visible light (in other words, sunlight); ii) it exhibits autonomous behavior, like motor proteins; iii) it does not generate waste products; iv) its operation can rely only on intramolecular processes, allowing in principle operation at the singlemolecule level; v) it can be driven at a frequency of about $1 \mathrm{kHz}$; vi) it works in mild environmental conditions (i.e. fluid solution at ambient temperature); and vii) it is stable for at least $10^{3}$ cycles.

A thorough computational investigation on $\mathbf{1}^{6+}$ has revealed ${ }^{[34]}$ that the rate-limiting step for the shuttling motion (process 4 in Fig. 2) could be related to the detachment of the $\mathrm{PF}_{6}{ }^{-}$counteranions from the station that has to receive the ring $\left(\mathrm{A}_{2}{ }^{2+}\right)$. If such a station were not hindered by anions, the shuttling motion would be almost barrierless and occur with a time constant as fast as $20 \mathrm{~ns}$ at $300 \mathrm{~K}$. Hence, the shuttling quantum yield could be substantially improved by adopting weakly coordinating counteranions for $\mathbf{1}^{6+}$, or by changing the solvent. The latter choice, however, would also affect the energetics and kinetics of the electron-transfer processes. Unfortunately, experiments in these directions are not easy because of difficulties related to counteranion exchange for $\mathbf{1}^{6+}$, and solubility issues. 
The molecular shuttle $\mathbf{1}^{6+}$ can also be operated, with a higher quantum yield, by a sacrificial mechanism ${ }^{[31]}$ based on the participation of external reducing (triethanolamine) and oxidizing (dioxygen) species, and by an intermolecular mechanism ${ }^{[32]}$ involving the kinetic assistance of an external electron relay (phenothiazine), which is not consumed. However, operation by the sacrificial mechanism does not afford an autonomous behavior and leads to consumption of chemical fuels and formation of waste products. On the other hand, the assistance by an electron relay affords autonomous operation in which only photons are consumed, but the mechanism is no longer based solely on intra-rotaxane processes.

We have also investigated systems related to $\mathbf{1}^{6+}$ in which either the position of the $\mathrm{A}_{1}{ }^{2+}$ and $\mathrm{A}_{2}{ }^{2+}$ stations is exchanged with respect to the $\mathrm{P}$ unit, ${ }^{[35]}$ a different $\mathrm{Ru}$ based moiety is employed, ${ }^{[36]}$ or the photosensitizer is connected noncovalently to an electron-accepting station. ${ }^{[37,38]}$

Recently, a second-generation molecular shuttle was designed and constructed. [39] The formula of rotaxane $2^{4+}$ and its operation scheme, which is similar to that of $\mathbf{1}^{6+}$ (Fig. 2), are shown in Fig. 3. The system is composed of a cyclobis(paraquat- $p$-phenylene) electron-accepting ring $\left(\mathrm{R}^{4+}\right)$ and a multicomponent dumbbell comprising a light-harvesting porphyrin $(\mathrm{P})$ which acts as an electron donor in the excited state, a $\mathrm{C}_{60}$ electron acceptor (A), and a tetrathiafulvalene (TTF, $\mathrm{D}_{1}$ ) and a dioxynaphthalene (DON, $\mathrm{D}_{2}$ ) electron-donating stations. In the stable translational isomer the electron-accepting ring, which is confined in the region of the dumbbell delimited by the two stoppers $T_{1}$ and $T_{2}$, encircles the better electron-donor TTF station. In solution, excitation of the porphyrin unit with visible light (process 1 in Fig. 3) should cause an electron transfer to $C_{60}$ (process 2); then, an electron shift from TTF to the oxidized porphyrin (process 4) should destabilize the original structure, causing the displacement of $\mathrm{R}^{4+}$ from $\mathrm{D}_{1}{ }^{+}$ to $\mathrm{D}_{2}$ (process 5). Subsequent back electron transfer (process 7) and macrocycle replacement (process 8 ) regenerate the starting isomer.

Rotaxane $\mathbf{2}^{4+}$ is expected to exhibit a better performance as a light-driven autonomous molecular shuttle compared with the first-generation prototype $\mathbf{1}^{6+}$ for the following three reasons. First, by using a triad approach, ${ }^{[40,41]}$ a relatively long lived charge-separated state should be obtained with a high efficiency. Second, the electrostatic repulsion between the photo-oxidized $\mathrm{D}_{1}{ }^{+}$station and the $\mathrm{R}^{4+}$ ring is expected to speed up the displacement of the latter (process 5 in Fig. 3). Third, the hampering effect of the counteranions discussed for $\mathbf{1}^{6+}$

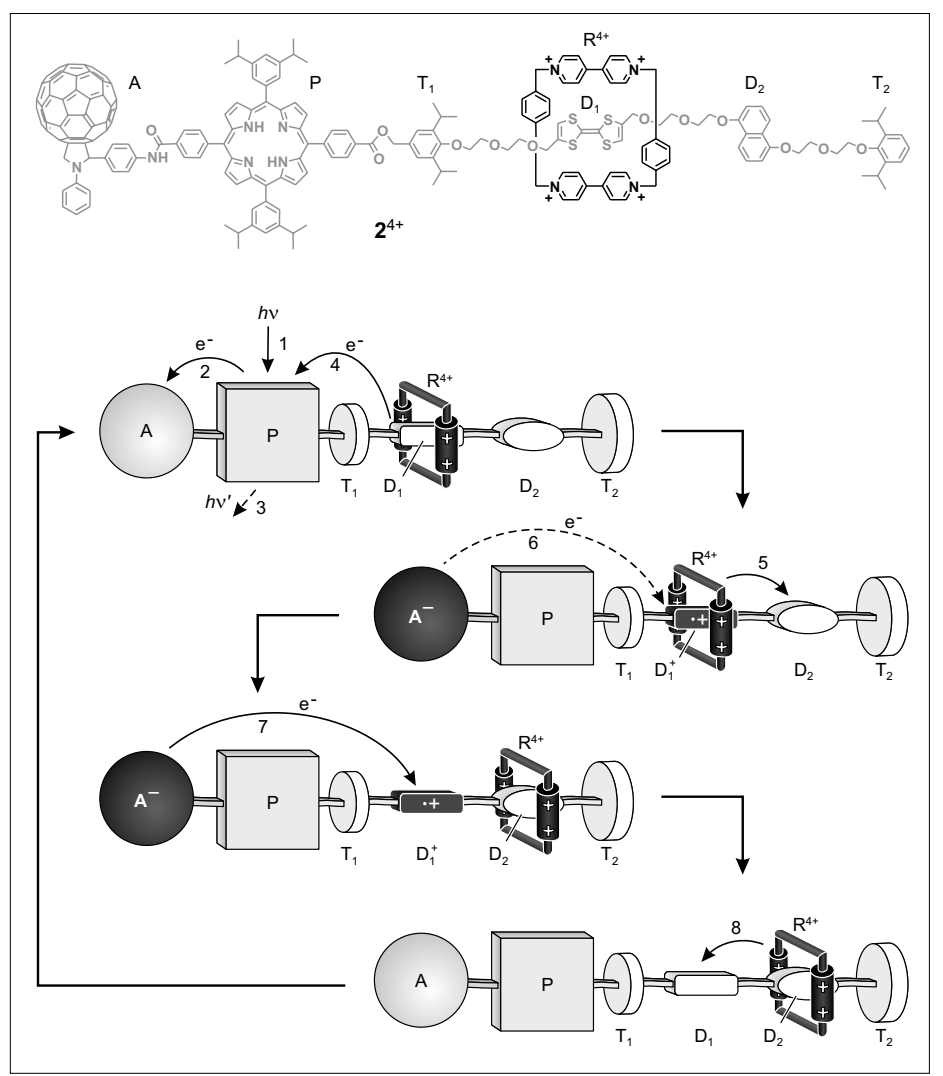

Fig. 3. Structure formula of rotaxane $2^{4+}$ and schematic representation of its operation as an autonomous molecular shuttle powered by visible light

is not expected to be dramatic in the case of $2^{4+}$ because the stations are originally uncharged.

UV-Vis-NIR absorption spectroscopic investigations and voltammetric experiments revealed ${ }^{[39]}$ remarkable electronic interactions between the various units of $2^{4+}$, pointing to the existence of folded conformations in solution. The TTF unit can be electrochemically oxidized only in a limited fraction of the rotaxane molecules. In these species, removal of one electron from the TTF unit causes the shuttling of the $\mathrm{R}^{4+}$ ring away from this station. Most likely, rotaxane $\mathbf{2}^{4+}$ occurs as conformations in which the TTF unit is buried inside a complex molecular structure and is therefore protected against oxidation performed by an electric potential applied externally. Such a behavior limits the efficiency for the operation of $2^{4+}$ as a redox-driven molecular shuttle. The possibility of achieving TTF oxidation by an electric potential generated internally through intramolecular photoinduced electron transfer is currently under investigation. In general terms, these results indicate that, as the structural complexity increases, the overall properties of the system cannot be easily rationalized solely on the basis of the type and sequence of the functional units incorporated in the molecular framework - that is, its 'primary' structure. Higher-level conformational effects, which are reminiscent of those related to the secondary and tertiary structure of biomolecules, ${ }^{[5]}$ have to be taken into consideration. The comprehension of these effects constitutes a stimulating scientific problem, and a necessary step for the design of novel artificial molecular devices and machines.

\subsection{Threading-Dethreading Motions Controlled by Photoin- duced Proton Transfer}

As shown by the examples described in the previous section, a modular construction of light-driven molecular machines pursued by integrating photoactive and mechanically switchable systems in a molecular assembly is a quite demanding task. In fact, the majority of artificial molecular machines reported so far ${ }^{[3,7-19]}$ are powered by chemical energy, most typically supplied by acid-base reactions. ${ }^{[42]}$ It would therefore be interesting to identify viable strategies for using light to operate 'stand alone' $\mathrm{pH}$-driven molecular machines. We showed recently ${ }^{[43]}$ that the acid-base controlled threading-dethreading of a pseudorotaxane in solution can be operated by light-induced intermolecular proton transfer with a photochromic switch. Studies on switchable pseudorotaxanes are important for the development of less trivial unimolecular machines based on rotaxanes, catenanes, and related interlocked compounds.

The calix[6]arene 3 (Fig. 4a) forms fairly stable pseudorotaxane complexes with 4,4'-bipyridinium derivatives in apolar 


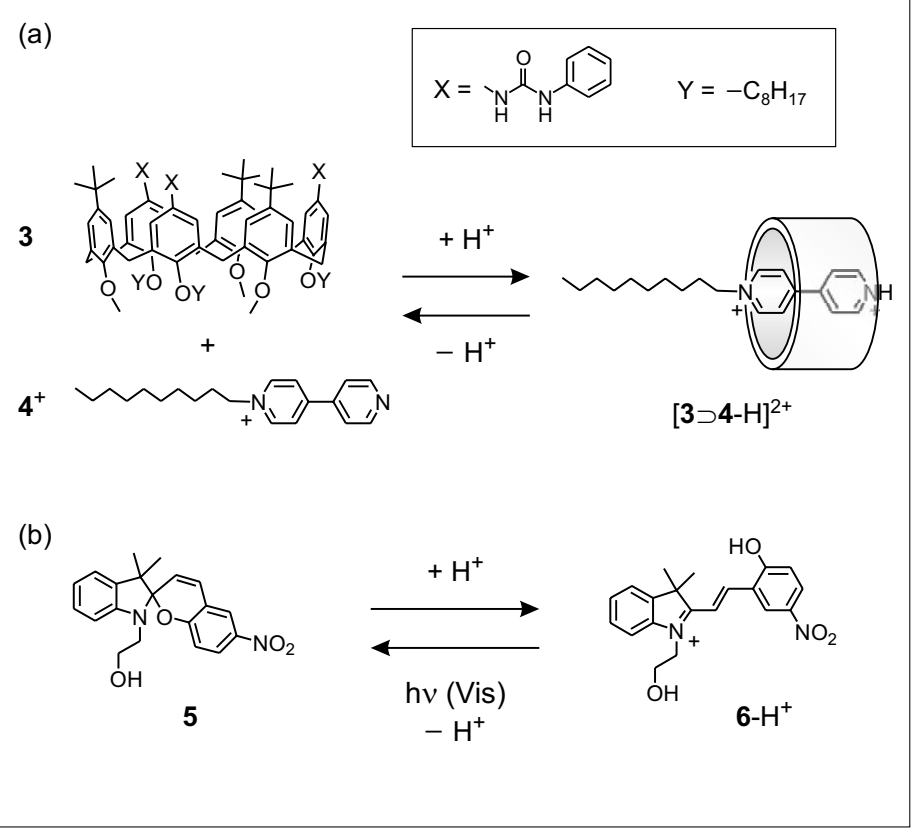

Fig. 4. (a) The acid-base controlled threading-dethreading of pseudorotaxane [3つ4-H] $]^{2+}$; (b) The acid-base and light controlled interconversion between spiropyran 5 and protonated merocyanine $6-\mathrm{H}^{+}$

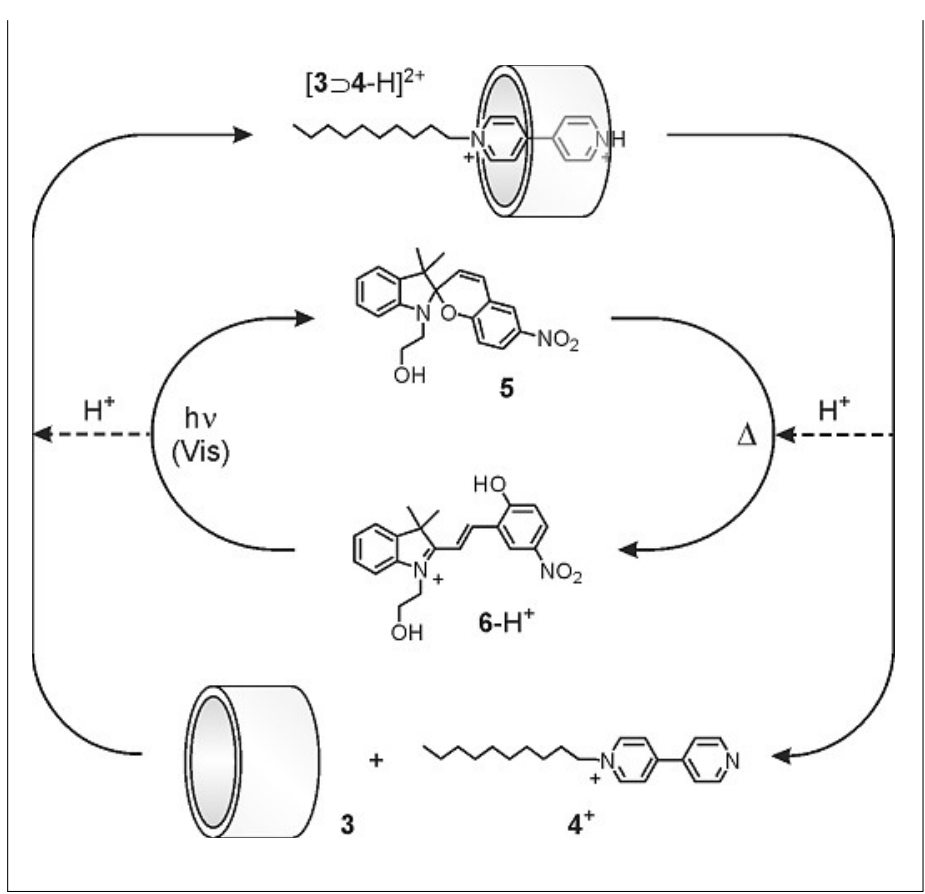

Fig. 5. Scheme for the control of threading-dethreading processes in pseudorotaxane $[3 \supset 4-H]^{2+}$ by means of photoinduced proton exchange with the photochromic system $5 / 6-\mathrm{H}^{+}$ solvents. ${ }^{[44]}$ Therefore, we envisaged that compound $4-\mathrm{H}^{2+}$, obtained by protonation of the pyridine nitrogen of the 4,4'-pyridylpyridinium thread-like $\mathbf{4}^{+}$(Fig. 4a), could thread into the cavity of $\mathbf{3}$ as well. In fact, spectrophotometric titrations and voltammetric experiments showed that a very stable pseudorotaxane complex is formed between 3 and $4-\mathrm{H}^{2+}$ in $\mathrm{CH}_{2} \mathrm{Cl}_{2}$. Deprotonation of $4-\mathrm{H}^{2+}$ with a base (e.g. tributylamine) in $\mathrm{CH}_{2} \mathrm{Cl}_{2}$ leads to dethreading of the pseudorotaxane (Fig. 4a).

In order to trigger the self-assembly and disassembly of this pseudorotaxane by light, we identified a species (Fig. 4b) that occurs in two forms, interconvertible into one another by light irradiation, exhibiting smaller and larger acid strength than that of $4-\mathrm{H}^{2+}$, respectively. ${ }^{[45,46]}$ In the presence of an acid, the colorless spiropyran $\mathbf{5}$ is converted into the yellow protonated merocyanine form 6- $\mathrm{H}^{+} .{ }^{[45]}$ Upon irradiation with visible light, 6- $\mathrm{H}^{+}$releases a proton, isomerizing back to 5 (Fig. 4b). [46]

We characterized this system by performing repeated photochemical and thermal equilibration experiments and using UV-vis absorption spectroscopy to monitor its state. ${ }^{[43]}$ The observed behavior can be interpreted in terms of the mechanism shown in Fig. 5. Starting from a mixture of the complex $[\mathbf{3} \supset \mathbf{4}-\mathrm{H}]^{2+}$ and $\mathbf{5}$ in a $1: 1$ ratio, a thermal proton transfer occurs from $4-\mathrm{H}^{2+}$ to the photochrome, yielding 6- $\mathrm{H}^{+}$and the deprotonated guest $\mathbf{4}^{+}$, which escapes from the cavity of $\mathbf{3}$. Subsequent light irradiation of $6-\mathrm{H}^{+}$in the visible re- gion causes an opposite proton transfer, converting $\mathbf{4}^{+}$into $4-\mathrm{H}^{2+}$; the latter species then rethreads into the calixarene macrocycle. Thermal equilibration-light irradiation cycles were performed on the same solution without loss of signal, showing that the overall switching process is reversible. As the reset of the system occurs thermally, its operation under continuous light irradiation can give rise to autonomous behavior. In practice, because of the large difference in the time scale of the dark and light parts of the cycle, the photostationary state is strongly displaced towards the $[3 \supset 4-H]^{2+} / 5$ mixture, unless irradiation is carried out with very low intensity and/or the temperature is increased. Nevertheless, such a behavior could be employed to implement a memory effect in the system. ${ }^{[47]}$ This system, although far from applications, is interesting because it provides a general principle for the operation of photoinactive acid-base controllable molecular machines with light.

\section{Conclusion and Perspectives}

The results described here show that compounds capable of performing large amplitude, non-trivial and controlled mechanical movements upon light stimulation can be obtained, with inspiration from natural systems, by utilizing careful incremental design strategies, the tools of modern synthetic chemistry, and the paradigms of supramolecular chemistry.
These achievements enable to devise future developments which are under investigation in our and other laboratories, namely: i) the design and construction of more sophisticated artificial molecular machines, showing complex motions and better performances in terms of stability, speed, switching, and so forth; ii) the use of such systems to do molecular-level tasks such as uptake-release, transportation, catalysis, and mechanical gating of molecular channels; and iii) the possibility of exploiting their logic behaviour for information processing at the molecular level and, in the long run, for the construction of chemical computers.

It is worth noting that the studies in solution of complicated chemical systems such as molecular machines and motors are of fundamental importance to understand their operation mechanisms. Moreover, for some use (e.g. drug delivery) molecular machines will have to work in liquid solution; in this regard, it should be recalled that motor proteins operate in - or in contact with - an aqueous solution. However, for applications in many fields of technology, artificial nanomachines have to be interfaced with the macroscopic world ${ }^{[48]}$ by ordering them in some way so that they can behave coherently (e.g. for exploiting collective behaviour ${ }^{[49]}$ ) and can be addressed in space. Indeed, new generations of molecular machines organized at interfaces or deposited on surfaces have started to appear. ${ }^{[50]}$

Apart from more or less futuristic applications, the study of motion at the molecu- 
lar level and the extension of the concept of motor and machine to the nanoscale are fascinating topics for basic research. Looking at molecular and supramolecular species from the viewpoint of functions with references to devices of the macroscopic world is indeed a very interesting exercise which introduces novel concepts into Chemistry as a scientific discipline.

\section{Acknowledgments}

We would like to thank Profs. V. Balzani and M. Venturi for their support, and all our collaborators and coworkers whose names appear in the reference list. In particular, we acknowledge a long lasting and fruitful cooperation with Prof. J. Fraser Stoddart and his group. Financial support from the EU (Biomach project), Ministero dell'Università e della Ricerca (PRIN 2006034123_003), Ministero degli Affari Esteri (DGPCC), Regione EmiliaRomagna (NANOFABER) and Università di Bologna (Progetto strategico CompReNDe) is gratefully acknowledged.

Received: February 7, 2008

[1] 'ITRS Roadmap for Semiconductors, 2007 Edition', available at $h t t p: / / w w w$. itrs.net (accessed April 2008).

[2] S. A. Edwards, 'The Nanotech Pioneers', Wiley-VCH, Weinheim, 2006.

[3] V.Balzani,A.Credi, M. Venturi, 'Molecular Devices and Machines - Concepts and Perspectives for the Nanoworld', WileyVCH, Weinheim, 2008.

[4] 'Molecular Motors', Ed. M. Schliwa, Wiley-VCH, Weinheim, 2003.

[5] D. S. Goodsell, 'Bionanotechnology Lessons from Nature', Wiley, Hoboken, 2004.

[6] R. A. L. Jones, 'Soft Machines Nanotechnology and Life', OUP, Oxford, 2005.

[7] V. Balzani, A. Credi, F. M. Raymo, J. F. Stoddart, Angew. Chem., Int. Ed. 2000, 39, 3348.

[8] A. H. Flood, R. J. A. Ramirez, W. Q. Deng, R. P. Muller, W. A. Goddard, J. F. Stoddart, Aust. J. Chem. 2004, 57, 301.

[9] Top. Curr. Chem. 2005, 262; special volume on Molecular Machines, Ed. T. R. Kelly.

[10] G. S. Kottas, L. I. Clarke, D. Horinek, J. Michl, Chem. Rev. 2005, 105, 1281.

[11] K. Kinbara, T. Aida, Chem. Rev. 2005, 105, 1377.

[12] F. C. Simmel, W. U. Dittmer, Small 2005 , $1,284$.

[13] Org. Biomol. Chem. 2006, 4; special issue on DNA-based Nanoarchitectures and Nanomachines, Ed. I. Willner.

[14] A. Credi, Aust. J. Chem. 2006, 59, 157.
[15] W. R. Browne, B. L. Feringa, Nat. Nanotech. 2006, 1, 25.

[16] E. R. Kay, D. A. Leigh, F. Zerbetto, Angew. Chem., Int. Ed. 2007, 46, 72.

[17] Adv. Funct. Mater. 2007, 17; special issue on Molecular Machines and Switches, Eds. A. Credi, H. Tian.

[18] B. Champin, P. Mobian, J.-P. Sauvage, Chem. Soc. Rev. 2007, 36, 358.

[19] A. Mateo-Alonso, D. M. Guldi, F. Paolucci, M. Prato, Angew. Chem., Int. Ed. 2007, 46, 8120 .

[20] V. Balzani, A. Credi, M. Venturi, Chem. Eur. J. 2008, 14, 26.

[21] N. Armaroli, V. Balzani, Angew. Chem. Int. Ed. 2007, 46, 52.

[22] G. Steinberg-Yfrach, J.-L. Rigaud, E. N. Durantini, A. L. Moore, D. Gust, T. A Moore, Nature 1998, 392, 479.

[23] 'Catenanes, rotaxanes and knots', Eds. J.-P. Sauvage, C. Dietrich-Buchecker, Wiley-VCH, Weinheim, 1999.

[24] 'Electron Transfer in Chemistry', Ed. V. Balzani, Wiley-VCH, Weinheim, 2001.

[25] R. Ballardini, V. Balzani, M. T. Gandolfi, L. Prodi, M. Venturi, D. Philp, H. G. Ricketts, J. F. Stoddart, Angew. Chem. Int. Ed. Engl. 1993, 32, 1301.

[26] P. R. Ashton, R. Ballardini, V. Balzani, S. E. Boyd, A. Credi, M. T. Gandolfi, M. Gómez-López, S. Iqbal, D. Philp, J. A. Preece, L. Prodi, H. G. Ricketts, J. F. Stoddart, M. S. Tolley, M. Venturi, A. J. P. White, D. J. Williams, Chem. Eur. J. 1997, 3,152 .

[27] A. C. Benniston, A. Harriman, D. S. Yufit Angew. Chem. Int. Ed. Engl. 1997, 36, 2356.

[28] P. R. Ashton, R. Ballardini, V. Balzani, E. C. Constable, A. Credi, O. Kocian, S. J. Langford, J. A: Preece, L. Prodi, E. R. Schofield, N. Spencer, J. F. Stoddart, S Wenger, Chem. Eur. J. 1998, 4, 2413.

[29] P. R. Ashton, V. Balzani, O. Kocian, L. Prodi, N. Spencer, J. F. Stoddart, J. Am. Chem. Soc. 1998, 120, 11190.

[30] S. Saha, E. Johansson, A. H. Flood, H.-R Tseng, J. I. Zink, J. F. Stoddart, Chem. Eur. J. 2005, 11, 6846.

[31] P. R. Ashton, R. Ballardini, V. Balzani, A. Credi, R. Dress, E. Ishow, C. J. Kleverlaan, O. Kocian, J. A. Preece, N. Spencer, J. F. Stoddart, M. Venturi, S. Wenger, Chem. Eur. J. 2000, 6, 3558.

[32] V. Balzani, M. Clemente-León, A. Credi, B. Ferrer, M. Venturi, A. H. Flood, J. F. Stoddart, Proc. Natl. Acad. Sci. USA 2006, 103, 1178

[33] For a related example of a photochemically driven molecular shuttle, see: A. M Brouwer, C. Frochot, F. G. Gatti, D. A. Leigh, L. Mottier, F. Paolucci, S. Roffia, G. W. H. Wurpel, Science 2001, 291, 2124.
[34] P. Raiteri, G. Bussi, C. S. Cucinotta, A. Credi, J. F. Stoddart, M. Parrinello, in press, DOI: 10.1002/anie.200705207.

[35] V. Balzani, M. Clemente-León, A. Credi, M. Semeraro, M. Venturi, H.-R. Tseng, S. Wenger, S. Saha, J. F. Stoddart, Aust. J. Chem. 2006, 59, 193.

[36] G. J. E. Davidson, S. J. Loeb, P. Passaniti, S. Silvi, A. Credi, Chem. Eur. J. 2006, 12, 3233.

[37] G. Rogez, B. Ferrer Ribera, A. Credi, R. Ballardini, M. T. Gandolfi, V. Balzani, Y. Liu, B. H. Northrop, J. F. Stoddart, J. Am. Chem. Soc. 2007, 129, 4633.

[38] B. Ferrer, G. Rogez, A. Credi, R. Ballardini, M. T. Gandolfi, V. Balzani, Y. Liu, H.-R. Tseng, J. F. Stoddart, Proc. Natl. Acad. Sci. USA 2006, 103, 18411.

[39] S. Saha, A. H. Flood, J. F. Stoddart, S. Impellizzeri, S. Silvi, M. Venturi, A. Credi, J. Am. Chem. Soc. 2007, 129, 12159.

[40] D. Gust, T. A. Moore, A. L. Moore, Acc. Chem. Res. 2001, 34, 40.

[41] V. Balzani, A. Credi, M. Venturi, ChemSusChem, 2008, 1, 26, and refs therein.

[42] J. D. Badjic, V. Balzani, A. Credi, S. Silvi, J. F. Stoddart, Science 2004, 303, 1845.

[43] S. Silvi, A. Arduini, A. Pochini, A. Secchi, M. Tomasulo, F. M. Raymo, M. Baroncini, A. Credi, J. Am. Chem. Soc. 2007, 129, 13378 .

[44] A. Credi, S. Dumas, S. Silvi, M. Venturi, A. Arduini, A. Pochini, A. Secchi, J. Org. Chem. 2004, 69, 5881.

[45] J. T. C. Wojtyk, A. Wasey, N.-N. Xiao, P. M. Kazmaier, S. Hoz, C. Yu, R. P. Lemieux, E. Buncel, J. Phys. Chem. A 2007, 111, 2511.

[46] F. M. Raymo, S. Giordani, A. J. P. White, D. J. Williams, J. Org. Chem. 2003, 68 4158.

[47] F. M. Raymo, R. J. Alvarado, S. Giordani, M. A. Cejas, J. Am. Chem. Soc. 2003, 125 , 2361.

[48] M. Clemente-León, A. Credi, M.-V. Martínez-Díaz, C. Mingotaud, J. F. Stoddart, Adv. Mater. 2006, 18, 1291, and references therein.

[49] Y. Liu, A. H. Flood, P. A. Bonvallett, S. A. Vignon, B. H. Northrop, H.-R. Tseng, J. O. Jeppesen, T. J. Huang, B. Brough, M. Baller, S. Magonov, S. D. Solares, W. A. Goddard, C. M. Ho, J. F. Stoddart, J. Am. Chem. Soc. 2005, 127, 9745.

[50] V. Balzani, A. Credi, M. Venturi, ChemPhysChem 2008, 9, 202. 